\title{
Systemic availability and pharmacokinetics of nebulised budesonide in preschool children
}

\author{
L Agertoft, A Andersen, E Weibull, S Pedersen
}

\begin{abstract}
Aim-To evaluate the systemic availability and basic pharmacokinetic parameters of budesonide after nebulisation and intravenous administration in preschool children with chronic asthma.

Methods-Plasma concentrations of budesonide were measured for three hours after an intravenous infusion of $125 \mu \mathrm{g}$ budesonide. The children then inhaled a nominal dose of $1 \mathrm{mg}$ budesonide through the mouthpiece of a Pari LC Jet Plus nebuliser connected to a Pari Master compressor, and the plasma concentrations of budesonide were measured for another six hours. The amount of budesonide inhaled by the patient ("dose to subject") was determined by subtracting from the amount of budesonide put into the nebuliser, the amount remaining in the nebuliser after nebulisation, the amount emitted to the ambient air (filter), and the amount found in the mouth rinsing water.

Results-Ten patients aged 3 to 6 years completed both the intravenous and the inhaled treatment. The mean dose to subject was $23 \%$ of the nominal dose. The systemic availability of budesonide was estimated to be $6.1 \%$ of the nominal dose (95\% confidence intervals (CI), $4.6 \%$ to $8.1 \%$ ) or $26.3 \%$ of the dose to subject $(95 \%$ CI, $20.3 \%$ to $34.1 \%)$. Budesonide clearance was $0.54 \mathrm{1} / \mathrm{min}$ (95\% CI, 0.46 to 0.62$)$, steady state volume of distribution 55 litres $(95 \% \mathrm{CI}, 45$ to 68$)$, and the terminal half life was 2.3 hours ( $95 \%$ CI, 2.0 to 2.6 ). Conclusions-Approximately $6 \%$ of the nominal dose $(26 \%$ of the dose to subject) reached the systemic circulation of young children after inhalation of nebulised budesonide. This is about half the systemic availability found in healthy adults using the same nebuliser.

(Arch Dis Child 1999;80:241-247)
\end{abstract}

Department of Paediatrics, Kolding Hospital, DK-6000

Kolding, Denmark

L Agertoft

A Andersen

$S$ Pedersen

Astra Draco AB, PO Box 34, S-221 00, Lund, Sweden

E Weibull

Correspondence to: Dr Agercroft.

Accepted 29 June 1998

Inhaled corticosteroids are normally administered to preschool children by a pressurised metered dose inhaler plus a spacer with a valve system or by a jet nebuliser. Only a few clinical dose finding studies have been conducted with budesonide delivered by nebuliser in these age groups. ${ }^{12}$ These studies found that the minimum effective dose required to control the disease in young children with moderate and severe asthma symptoms varied markedly from patient to patient, the average daily nominal dose being $1 \mathrm{mg}^{2}{ }^{3}$ Considering the ages and body weights of preschool children, such a dose seems quite high compared with the recommendations for adults and the dose required when a pressurised metered dose inhaler with a spacer is used for the administration of budesonide. For these reasons some paediatricians have raised safety concerns about nebulised budesonide treatment. However, because of differences between children and adults in airway calibre and anatomy of the upper airways, the inspiratory air flow dynamics of children might be quite different from those of adults. Therefore, conclusions from adult studies on lung deposition and the systemic bioavailability of inhaled budesonide should be extrapolated to young children with great caution. Indeed, nothing is known about the pharmacokinetics of budesonide in preschool children or the percentage of the nominal dose that becomes systemically available after nebulisation.

The aim of our study was to assess the absolute systemic availability and basic pharmacokinetic parameters of budesonide given as a nebulised suspension in young children via a widely used jet nebuliser, Pari LC Jet Plus connected to a Pari Master compressor (PARI $\mathrm{GmbH}$, Starnberg, Germany). In addition, the method of assessing inhaled dose and nebuliser output in vivo by inserting filters to the inspiratory and expiratory outlets was evaluated.

\section{Patients and methods}

Children aged 3 to 6 years with chronic asthma requiring regular treatment with inhaled budesonide from a pressurised metered dose inhaler with a spacer were included in the study. All patients had to demonstrate a correct inhalation technique with the Pari LC Jet Plus nebuliser equipped with a mouthpiece. The study was performed in accordance with the Declaration of Helsinki. Written and verbal informed consent was obtained from the parents of all children before any study related procedure was conducted.

The design was an open, single centre, pharmacokinetic study consisting of three visits to the clinic. Visits 1 and 2 were separated by one to two weeks and visits 2 and 3 by no more than one week. Two treatments were given: an intravenous infusion of budesonide and a single inhaled dose of nebulised budesonide, both at visit 3. A semi-simultaneous design was usedthat is, the two administrations were given in a fixed order with the inhaled dose given three hours after the start of the infusion. 
VISIT 1 (TRAINING VISIT)

The patient was instructed and trained in the correct use of the nebuliser using placebo. Correct use was considered to be tidal breathing without any pauses or leakage around the mouthpiece. A nose clip was worn during the inhalation. When the child had a correct inhalation technique, he/she was given a nebuliser with a compressor and placebo for further training at home before the next visit.

VISIT 2 (FILTER INHALATION)

The patient's inhalation technique was checked (placebo). This was followed by an inhalation with filters (filter inhalation): an unused Pari LC Jet Plus nebuliser connected to a Pari Master compressor was equipped with an inspiratory filter between the nebuliser and the mouthpiece and an expiratory filter attached to the expiratory outlet. The amount of drug deposited on the inspiratory filter is assumed to be a measure of the amount of drug inhaled by the patient while the amount on the expiratory filter is considered to be a measure of the amount of drug emitted to the ambient air during nebulisation. The nebuliser was charged with $2 \mathrm{ml} \quad 0.5 \mathrm{mg} / \mathrm{ml}$ budesonide suspension and the patient inhaled as described under visit 1 . Nebulisation time was five minutes. The nebuliser was tapped gently during nebulisation and it was ensured that the patient had a correct inhalation technique. The nebuliser was weighed before and after charging and after nebulisation. The filters were stored in dark plastic bags at room temperature until analysis for budesonide content by a liquid chromatography method.

At the end of the visit, the nebuliser was dismantled, thoroughly washed with ethanol, left to dry, and then reassembled, ready to be used again by the same patient at visit 3 .

VISIT 3 (DRUG INHALATION)

Inhaled budesonide was discontinued two days before visit 3 . On arrival at the clinic, indwelling catheters (Venflon; Becton Dickinson BOC Ohmeda AB, Helsinborg, Sweden) were inserted in both arms after pretreatment with EMLA anaesthetic patches (Astra AB, Södertälje, Sweden). One arm was used for intravenous infusion of budesonide and the other for blood sampling.

A budesonide solution ( $20 \mathrm{ml}$ of $6.25 \mu \mathrm{g} / \mathrm{ml}$, total dose $125 \mu \mathrm{g}$ ) was infused manually at a constant rate over 10 minutes. The infusion was followed by an injection of $5 \mathrm{ml}$ sterile saline to rinse the catheter before it was removed. A sample of the infused budesonide solution was frozen for later analysis of budesonide concentration. The syringe was weighed before and after infusion to obtain the infused weight which, together with the measured concentration and the density of the infused solution, was used to calculate the exact amount of budesonide given.

Three hours after the start of the infusion, nebulised budesonide was administered in exactly the same way as during filter inhalation at visit 2 , except that an inspiratory filter was not used and the nebuliser was not weighed.
The patients rinsed their mouths twice with $10 \mathrm{ml}$ tap water directly after nebulisation and the rinsing water was collected for later analysis for budesonide content. The budesonide content in the intravenous solution, on the expiratory filter, in the ampoule, in the nebuliser, and in the mouth rinsing water was determined by a liquid chromatography method. Rigorous precautions were taken to avoid contamination of the plasma samples with budesonide from the nebulisation.

Blood samples $(4-5 \mathrm{ml})$ were drawn before the start of infusion (0), at the end of infusion (10 minutes), at $15,30,60,90$, and 120 minutes after the start of infusion and at 180 minutes after the start of infusion (immediately before inhalation). In addition, samples were taken at the end of inhalation and at 20, 40, 80, $160,240,330$, and 360 minutes after the start of inhalation. The plasma samples were stored frozen until analysis for budesonide content. The total sampling time was nine hours and the total blood volume taken from each child was approximately $70 \mathrm{ml}$.

If a patient refused to have the second Venflon inserted for intravenous administration, the absolute systemic availability of budesonide could not be determined. However, the inhalation and subsequent blood sampling could still be carried out.

The plasma concentration of the sum of the two budesonide epimers (22R and 22S) was determined by a liquid chromatography plus mass spectrometry method. The lower limit of quantification was $0.025 \mathrm{nmol} / \mathrm{l}$. The between assay coefficient of variation $(\mathrm{CV})$ in control samples run in parallel with the study samples was $16.4 \%$ at $0.025 \mathrm{nmol} / 1$ and $2.9 \%$ at $4.0 \mathrm{nmol} / 1$.

IN VITRO CHARACTERISTICS OF THE NEBULISER Pari LC Jet Plus delivers a dose of budesonide, measured in vitro with filters, of about $23 \%$ of the nominal dose when charged with $2 \mathrm{ml}$ $0.5 \mathrm{mg} / \mathrm{ml}$ of budesonide. The droplet size, measured as mass median diameter with a Malvern Mastersizer X (Malvern, Worcestershire, UK), is $5 \mu \mathrm{m}$. When charged with $2 \mathrm{ml}$ of budesonide suspension, the nebuliser normally runs to dryness within five minutes.

PHARMACOKINETIC ANALYSIS AND STATISTICAI METHODS

The dose inhaled by the patient during nebulisation was assessed at visits 2 and 3 .

\section{Filter inhalation}

The amount of budesonide recovered from the inspiratory (I) and expiratory (E) filters was used to estimate the inhaled dose, the total output from the nebuliser $(\mathrm{I}+\mathrm{E})$, and the inhaled dose in percentage of the total output $(\mathrm{I} /(\mathrm{I}+\mathrm{E}))$.

\section{Drug inhalation}

The "dose to subject" was calculated by subtracting from the assayed batch dose the sum of the amounts of budesonide recovered after nebulisation from the ampoule, the 
Table 1 Measurement of inhaled budesonide dose, budesonide dose on expiratory filter, and inhaled dose in \% of total output in 12 preschool children by two different methods (filter and drug inhalation)

\begin{tabular}{|c|c|c|c|c|c|c|}
\hline \multirow[b]{2}{*}{ Number } & \multicolumn{2}{|l|}{ Inhaled dose $(\mu \mathrm{g})$} & \multicolumn{2}{|c|}{ Dose on expiratory filter $(\mu g)$} & \multicolumn{2}{|c|}{ Inhaled dose in $\%$ of total output } \\
\hline & Filter inhalation & Drug inhalation & Filter inhalation & Drug inhalation & Filter inhalation & Drug inhalation \\
\hline 1 & 214 & 221 & 198 & 215 & 52 & 51 \\
\hline 2 & 237 & 270 & 177 & 260 & 57 & 51 \\
\hline 3 & 231 & 274 & 201 & 256 & 53 & 52 \\
\hline 5 & 239 & 230 & 206 & 234 & 54 & 50 \\
\hline 6 & 250 & 224 & 166 & 225 & 60 & 50 \\
\hline 7 & 239 & 198 & 176 & 202 & 58 & 49 \\
\hline 8 & 229 & 251 & 170 & 217 & 57 & 54 \\
\hline 9 & 266 & 249 & 177 & 286 & 60 & 46 \\
\hline 10 & 253 & 199 & 166 & 254 & 60 & 44 \\
\hline 11 & 293 & 261 & 161 & 186 & 65 & 58 \\
\hline 12 & 275 & 242 & 176 & 241 & 61 & 50 \\
\hline 13 & 235 & 232 & 211 & 245 & 53 & 49 \\
\hline G mean & 245.9 & 235.9 & 181.4 & 233.6 & 57.4 & 50.1 \\
\hline CV (\%) & 8.7 & 10.7 & 9.3 & 12.0 & 6.8 & 7.0 \\
\hline
\end{tabular}

G mean; geometric mean; CV, coefficient of variation.

expiratory filter, the nebuliser with mouthpiece and connecting tubes, and the mouth rinsing water.

The inhaled dose was calculated as the sum of the dose to subject and the amount of drug recovered from the mouth rinsing water.

The total output from the nebuliser was calculated as dose to subject plus the amounts of budesonide recovered from the mouth rinsing water and expiratory filter.

Doses and ratios between doses or amounts from filter and drug inhalations were described with the geometric mean and 95\% confidence limits using the $t$ distribution. The nominal dose was defined as the dose written on the package label.

NON-PARAMETRIC PHARMACOKINETIC ANALYSES Because the half life of the last observable phase after intravenous administration was found to be shorter than the terminal half life seen after inhalation, different analyses based on different pharmacokinetic assumptions were possible. In the primary analysis, the terminal half life after intravenous administration was assumed to be identical to the terminal half life seen after inhalation (identical half lives). Thus, the terminal elimination rate constant, $\mathrm{k}_{\mathrm{el}}$ was estimated from the plasma concentrations after inhalation by mathematically fitting a straight line to the last plasma measurements (ln(concentration) $v$ time) using linear regression. The intravenous and the inhalation curves were then separated from each other by assuming that the terminal phase for the intravenous dose was reached when the inhalation started, three hours after the intravenous dose. The extrapolated concentrations from the intravenous curve were subtracted from the measured concentrations after inhalation to obtain a plasma concentration curve for inhalation.

The pharmacokinetic parameters calculated using non-parametric methods were described with means and $95 \%$ confidence limits for the mean calculated using the $t$ distribution. Geometric means were used for $\mathrm{T}_{1 / 2}$ (terminal half life), $\mathrm{Cl}$ (total clearance), $\mathrm{V}_{\mathrm{d}}$ (volume of distribution during terminal phase), $\mathrm{V}_{\mathrm{ss}}$ (volume of distribution at steady state), $\mathrm{F}_{\mathrm{DTS}}^{\mathrm{ss}}$ (systemic bioavailability in percentage of dose to sub- ject), and $F$ (systemic bioavailability after inhalation in percentage of nominal dose).

In the secondary analysis, the last observable phase after the intravenous administration was assumed to be the terminal elimination phase after an intravenous dose (different half lives) and the curves were separated using this assumption. This may be interpreted as an absorption rate limited elimination for nebulised budesonide (a flip flop phenomenon). This analysis might also provide an upper limit for the systemic availability if there is no flip flop, but the terminal phase after intravenous administration starts later than three hours after dose.

PARAMETRIC PHARMACOKINETIC ANALYSIS

A pharmacokinetic model was fitted to the plasma concentration data using a non-linear mixed effects model approach. The population parameters were estimated using the VoneshCarter algorithm ${ }^{4}$ and approximate $95 \%$ confidence limits calculated.

A three exponential model was chosen for the intravenous administration because two clear phases were seen after the intravenous dose and a third, slower phase, was seen after the inhalation.

\section{Results}

Thirteen children aged 3 to 6 years were included in our study. Ten patients completed both intravenous and inhaled treatment. One child received only inhaled treatment because of problems with the intravenous administration. Two children were withdrawn because of lack of cooperation and problems with blood sampling, but the inhalation data from one of these children could be used, leaving 12 children for comparison between filter and drug inhalation. The mean age of the eight boys and two girls who completed both budesonide administrations on visit 3 was 4.7 years (range, 3-6). Their mean height was $109 \mathrm{~cm}$ (range, 95.5-121.0); mean weight was $18.4 \mathrm{~kg}$ (range, 15.0-25.5). No adverse events were reported in our study.

FILTER INHALATIONS (TABLE 1)

A mean of $246 \mu \mathrm{g}$, or $25 \%$ of the nominal dose, was recovered from the inspiratory filter. This 


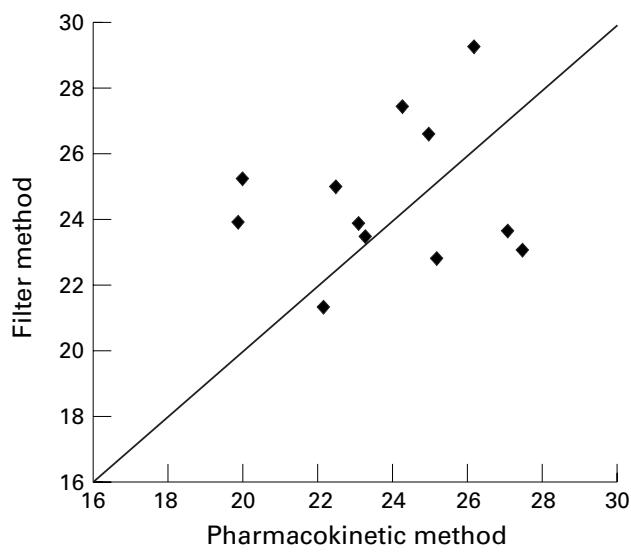

Figure 1 Correlation between estimates of inhaled dose (\% of nominal) from filter inhalation and drug inhalation of budesonide from a Pari LC Fet Plus nebuliser in 12 preschool children with asthma.

corresponds to $57 \%$ of the total nebuliser output, which was $428 \mu \mathrm{g}$, or $43 \%$ of the nominal dose. The between-patient variation in these data was low with CVs below $10 \%$. Of the charged weight, $70 \%$ was nebulised.

DRUG INHALATION (TABLE 1)

The total output from the nebuliser (inhaled dose + drug on expiratory filter) was $471 \mu \mathrm{g}$, or $47 \%$ of the nominal dose. The mean inhaled dose (dose to subject + amount in mouth rinsing water) was $236 \mu \mathrm{g}$ or $24 \%$ of the nominal dose $(50 \%$ of total nebuliser output). The mean dose to subject was $232 \mu \mathrm{g}$ (23\% of nominal dose). The between patient variation in all these estimates was of the same magnitude as for the corresponding estimates from the filter inhalation (table 1).

COMPARISON BETWEEN FILTER INHALATION AND DRUG INHALATION

In general, there was good agreement between the methods of assessing the inhaled dose during nebulisation (table 1 ; fig 1 ). The mean ratio between the amount on the inspiratory filter and inhaled dose after drug inhalation was $104 \%$ ( $95 \%$ CI, $96 \%$ to $113 \%$ ). The mean ratio between the total output from the nebuliser after filter and drug inhalation was $91 \%(95 \%$ CI, $86 \%$ to $96 \%)$; there was a significantly lower total output after filter inhalation. The mean ratio between the amounts found on the expiratory filters after filter and drug inhalation was $78 \%$ ( $95 \% \mathrm{CI}, 71 \%$ to $84 \%$ ); there was a significantly lower amount

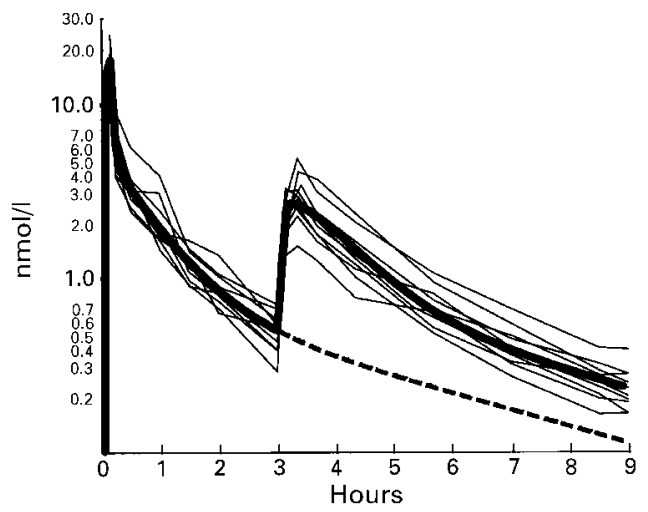

Figure 2 Individual (thin lines) and estimated mean (bold and bold dashed lines) budesonide plasma concentration curves (log scale) in 10 preschool children after intravenous infusion of $117 \mu \mathrm{g}$ budesonide and inhalation of $1000 \mu \mathrm{g}$ budesonide from a Pari LC Fet Plus nebuliser. Mean curve was obtained from the parametric analysis with a three exponential model.

on the expiratory filter after filter inhalation. Thus, the lower total output after filter inhalation was mainly a result of a lower amount on the expiratory filter after this inhalation.

PHARMACOKINETIC ANALYSIS

The administered intravenous budesonide dose ranged from 114 to $120 \mu \mathrm{g}$ (mean, $117 \mu \mathrm{g})$ and the dose to subject ranged from 192 to $273 \mu \mathrm{g}$ (geometric mean, $232 \mu \mathrm{g}$ ). All plasma samples after drug administration had concentrations above the limit of quantification. The individual plasma profiles after the intravenous and inhaled doses were very consistent in shape (fig 2). The half life of the last observable phase after intravenous administration was shorter than the terminal half life seen after inhalation for all patients.

Table 2 gives the mean pharmacokinetic parameters and $95 \%$ confidence limits calculated from the primary and secondary nonparametric analyses and from the three exponential model. Clearance and systemic availability in percentage of nominal dose or percentage of dose to subject were very similar in the three analyses, whereas half lives and volumes of distribution were, as expected, somewhat lower in the non-parametric analysis assuming different half lives.

SCALED PARAMETERS AND AGE DEPENDENCE To enable comparison of clearance and volumes of distribution with the values obtained in adults in a previous study, ${ }^{5}$ these parameters

Table 2 Estimated pharmacokinetic parameters of budesonide in 10 preschool children by: a non-parametric evaluation assuming identical terminal half lives after intravenous administration and inhalation; a non-parametric evaluation assuming different terminal half lives after intravenous administration and inhalation; and by a three exponential model

\begin{tabular}{|c|c|c|c|c|c|c|}
\hline & \multicolumn{2}{|c|}{ Identical half lives } & \multicolumn{2}{|c|}{ Different half lives } & \multicolumn{2}{|c|}{ Three exponential analysis } \\
\hline & Mean & $95 \% C I$ & Mean & $95 \% C I$ & Mean & $95 \% C I$ \\
\hline $\mathrm{Cl}(\mathrm{ml} / \mathrm{min})$ & 536 & (461 to 623 ) & 592 & (508 to 691$)$ & 504 & (436 to 576 ) \\
\hline $\mathrm{V}_{\mathrm{d}}$ (litres) & 105 & (91 to 122$)$ & 57 & (48 to 68 ) & 139 & (102 to 186$)$ \\
\hline $\mathrm{V}_{\mathrm{ss}}$ (litres) & 55 & (45 to 68 ) & 38 & (31 to 48 ) & 64 & (51 to 78 ) \\
\hline $\mathrm{T}_{1 / 2}$ (hours) & 2.3 & (2.0 to 2.6$)$ & 1.1 & (0.9 to 1.3$)$ & 3.2 & (2.3 to 4.3 ) \\
\hline $\mathrm{F}_{\mathrm{DTS}}(\%)$ & 26.3 & (20.3 to 34.1$)$ & 34.7 & (28.7 to 41.9$)$ & 24.2 & (19.1 to 30.6$)$ \\
\hline $\mathrm{F}(\%)$ & 6.1 & $(4.6$ to 8.1$)$ & 8.1 & (6.6 to 9.9 ) & 5.6 & ( 4.5 to 7.1 ) \\
\hline
\end{tabular}

$\mathrm{Cl}$, total clearance; $\mathrm{V}_{\mathrm{d}}$, volume of distribution in terminal phase; $\mathrm{V}_{\mathrm{ss}}$, volume of distribution at steady state; $\mathrm{F}_{\mathrm{DTS}}$, systemic bioavailability in $\%$ of dose to subject; $F$, systemic bioavailability in $\%$ of nominal dose. 
Table 3 Scaled pharmacokinetic parameters of budesonide in 10 preschool children estimated in a non-parametric evaluation assuming identical or different terminal half lives after intravenous infusion and inhalation of budesonide

\begin{tabular}{|c|c|c|c|c|}
\hline & \multicolumn{2}{|c|}{ Identical terminal half lives } & \multicolumn{2}{|c|}{ Different terminal half lives } \\
\hline & $\begin{array}{l}\text { Mean } \\
\text { (per kg) }\end{array}$ & $\begin{array}{l}\text { Mean } \\
\text { (scaled to } 70 \mathrm{~kg} \text { ) }\end{array}$ & $\begin{array}{l}\text { Mean } \\
\text { (per kg) }\end{array}$ & $\begin{array}{l}\text { Mean } \\
\text { (scaled to } 70 \mathrm{~kg} \text { ) }\end{array}$ \\
\hline $\mathrm{Cl}(\mathrm{ml} / \mathrm{min})$ & 29.8 & 1490 & 32.9 & 1645 \\
\hline $\mathrm{V}_{\mathrm{d}}$ (litres) & 5.9 & 411 & 3.2 & 225 \\
\hline $\mathrm{V}_{\mathrm{ss}}$ (litres) & 3.1 & 216 & 2.2 & 151 \\
\hline
\end{tabular}

$\mathrm{Cl}$, total clearance; $\mathrm{V}_{\mathrm{d}}$, volume of distribution in terminal phase; $\mathrm{V}_{\mathrm{ss}}$, volume of distribution at steady state.

were expressed per kg body weight (table 3). In addition, clearance was scaled to a $70 \mathrm{~kg}$ subject by using weight ${ }^{0.75}$ as an adjustment factor instead of simply scaling by weight ${ }^{1.0,6}$. Volumes were scaled to a $70 \mathrm{~kg}$ subject by weight $^{1.0}$ only. Table 3 gives a summary of the scaled parameters from the primary nonparametric analysis, assuming identical terminal half lives after infusion and inhalation. In addition, the parameters from non-parametric analysis assuming different terminal half lives after intravenous administration and inhalation are given for comparison. No age dependence was found for any of the pharmacokinetic parameters or inhaled dose, as assessed by scatter plots.

\section{Discussion}

Our study provided important information about the systemic availability of budesonide in young children after nebulisation. A systemic availability around $6 \%$ is about half the value previously found in healthy adults using the same nebuliser alone (13\%) (E Bondesson, personal communication, 1996) or with a Spira synchroniser $(15 \%) .{ }^{7}$ If the systemic availability is related to the dose to subject instead, the difference between children and adults seems even more pronounced: $26 \%$ in children and $63 \%$ (synchroniser) and $72 \%$ (no synchroniser) in adults. Lung deposition could be estimated to be around $18 \%$ of dose to subject in our children. This should be compared with estimated lung depositions of 58\% (synchroniser) and $70 \%$ (no synchroniser) in the two adult studies. Furthermore, the deposition in the oropharynx was estimated to be $\sim 82 \%$ of dose to subject in young children compared with only $42 \%$ (synchroniser) and $30 \%$ (no synchroniser) in adults. This indicates a very different deposition pattern in young children and adults, which is probably caused by the smaller dimensions of the oropharynx and larynx in young children. These findings emphasise that conclusions from adult deposition studies cannot be extrapolated to children. The studies must be conducted in the age groups in which the inhaler is going to be used.

When the clinical implications of these results are discussed it must be remembered that the standardised and very controlled study conditions differed greatly from the day to day clinical treatment situation. In day to day treatment, the variability in inhaled dose is likely to be higher and the lung deposition lower. ${ }^{89}$ In these age groups, use of a face mask is often associated with inhalation through the nose,

\section{Key messages}

- Approximately $6 \%$ of the nominal dose of budesonide ( $26 \%$ of dose to subject) reaches the systemic circulation of young children after inhalation from a Pari LC Jet Plus nebuliser

- Deposition of drug in the intrapulmonary airways seems to be much lower in young children than in adults using the same nebuliser

- Budesonide clearance/kg body weight is higher in young children than in adults

- The low systemic availability in combination with a higher clearance/kg body weight in young children means that these age groups can use the same nebulised budesonide dose as adults without an increased risk of unwanted systemic effects. Therefore, dosing of nebulised budesonide in $\mathrm{mg} / \mathrm{kg}$ body weight to reduce the risk of unwanted systemic effects is not warranted

- A filter inhalation accurately assesses the inhaled dose of budesonide in children using a nebuliser. However, in the individual patient it is only a crude surrogate marker of the systemic availability and dose of budesonide deposited in the intrapulmonary airways

which has been shown to reduce the dose deposited in the intrapulmonary airways by more than $50 \%$ compared with oral inhalation. ${ }^{810}$ Therefore, the estimate of lung deposition in our study is probably close to the maximum achievable with the nebuliser used. The use of a face mask is likely to reduce the therapeutic ratio (clinical effect/systemic effect ratio), whereas its influence on the total systemic effect is unpredictable.

There did not seem to be any association between the various pharmacokinetic parameters or inhaled dose and age, perhaps because the age range was too narrow. No other studies have assessed the possible age dependency of the various pharmacokinetic parameters of budesonide. Some studies have found age dependent lung deposition or drug delivery to the patient with various inhalation devices, ${ }^{11}{ }^{12}$ whereas others have found lung deposition to be independent of age. ${ }^{8} 9$

Pharmacokinetic data on intravenous budesonide in children are sparse, so it is difficult to make direct comparisons of our data with the findings of others. In our study, mean clearance was estimated to be around $30 \mathrm{ml} /$ $\mathrm{min} / \mathrm{kg}$, which is $\sim 50 \%$ higher than in healthy adults. ${ }^{5}$ This is in agreement with the findings in older children. ${ }^{13}$ Volume of distribution $/ \mathrm{kg}$ body weight in steady state was about $15 \%$ higher than in adults, ${ }^{5}$ whereas the terminal half life after inhalation (2.3 hours) seemed to be within the range of the terminal half life after intravenous administration in adults. In contrast, the half life after intravenous administration (1.2 hours) was much shorter than in 
adults. This could be a result of the study design, where the intravenous curve was followed for only three hours, and thus might have been truncated before the terminal phase was entered. These findings emphasise that comparisons between studies with different sampling times and designs should be made with caution, because the results may vary with the methods used.

A semi-simultaneous design was preferred to administrations on separate days because of the reduction in intra-individual variation, ${ }^{14}$ a lower number of vein punctures, fewer blood samples, a shorter sampling period, and an increased chance of good compliance and measurable plasma concentrations. It has been shown previously that the systemic availability of terbutaline could be estimated as accurately and precisely with this method as with the stable isotope method. ${ }^{15}$ In our study, however, the different half lives seen after intravenous administration and inhalation made several interpretations possible. Either the terminal half lives after intravenous administration and inhalation are identical and the intravenous curve is truncated before entering the terminal phase, or there is a difference in half lives as a result of an absorption rate limited elimination of inhaled drug. An absorption rate limited elimination of inhaled budesonide was considered an unlikely explanation for the difference in half lives, because no such observations have been made in adult pharmacokinetic studies with Turbuhaler or pressurised metered dose inhalers. ${ }^{5}$ However, the estimates of systemic availability and clearance from all three different analyses were in good agreement with mean availabilities in the range of $6-8 \%$ and mean clearances of $0.5-0.6 \mathrm{l} / \mathrm{min}$. This suggests that our conclusions are reasonably robust and not heavily dependent upon the choice of pharmacokinetic model.

Young children often use nebulised doses of budesonide that are in the same dose range as those used by adults. This causes great concern among many paediatricians and it is often suggested that nebulised drugs should be scaled to young children by dosing in $\mathrm{mg} / \mathrm{kg}$ to reduce the risk of systemic side effects. The appropriateness of this suggestion has not been thoroughly validated. However, our study allowed some assessments of this.

The systemic exposure of drug measured as the area under the plasma concentration $v$ time curve (AUC) is normally thought to reflect the systemic activity of the treatment. Therefore, it was interesting that the AUC/mg nominal dose in our study was very similar to the AUC/mg nominal dose measured in adults using the same nebuliser $(4.6$ and $3.9 \mathrm{nmol} / 1 \times$ hour $/ \mathrm{mg}$, respectively) (E Bondesson, personal communication, 1996). The same was the case if the calculation was done as AUC/mg dose to subject (19.8 for children and $21.0 \mathrm{nmol} / 1 \times$ hour/mg for adults). This strongly suggests that the systemic effects after inhalation of the same nominal dose of budesonide from a Pari nebuliser will be very similar in young children and adults, despite the marked difference in size. Thus, these results do not support scaling of the dose in $\mathrm{mg} / \mathrm{kg}$ to limit systemic effects.

Nebulisation time was fixed to five minutes because in vitro tests before the study showed this to be long enough to run the nebulisers to dryness. A fixed time end point also avoided the difficult judgment of "dryness" from the sputtering sound of the nebuliser. In our study, $70 \%$ of the charged weight was lost during nebulisation, suggesting that a nebulisation time of five minutes is sufficient to run the nebulisers to dryness in vivo when a $2 \mathrm{ml}$ charge is used. Therefore, we do not believe that this affected the conclusions of the study.

Filter studies are often used as surrogates for determination of the inhaled dose and lung deposition of drug. ${ }^{11}{ }^{12}{ }^{16-19}$ However, the filter method has not yet been validated in young children with asthma. In our study, there was excellent agreement between the two ways of assessing the inhaled dose. This is in accordance with the results of a study in adults assessing deposition of radiolabelled pentamidine in the intrapulmonary airways. ${ }^{20}{ }^{21}$ However, the poor correlation between inhaled dose and systemic availability in our study indicates that important factors other than inhaled dose influence the deposition of nebulised budesonide in the intrapulmonary airways. Our study does not allow any conclusion about which factors this could be.

The amount of drug on the expiratory filter and total drug output were slightly lower for the filter inhalation compared with the drug inhalation. The only difference between the two experimental set ups was the absence of an inspiratory filter during drug inhalation, suggesting that the introduction of an inspiratory filter might have caused the difference. This suggestion is in accordance with the findings of other studies. ${ }^{31622} 23$ The reason for this change is not clear. It might be a result of the increased dead space in the nebuliser or to the somewhat higher resistance caused by the filter. This should be remembered when the findings of filter studies are evaluated.

1 Ilangovan P, Pedersen S, Godfrey S, et al. Treatment of severe steroid dependent preschool asthma with nebulised budesonide suspension. Arch Dis Child 1993;68:356-9.

2 Wennergren G, Nordvall SL, Hedlin G, et al. Nebulized budesonide for the treatment of moderate to severe asthma in infants and toddlers. Acta Paediatr 1996;85:183-9.

3 Vikre-J $\not$ rgensen J, Agertoft L, Pedersen S. Dose titration of nebulized budesonide in young children. Pediatr Pulmonol 1997;23:270-7

4 Vonesh EF, Carter RL. Mixed effect models for unbalanced repeated measures. Biometrics 1992;48:1-18.

5 Thorsson L, Edsbäcker S, Conradson TB. Lung deposition of budesonide from Turbuhaler is twice that from a pressurized metered dose inhaler (MDI). Eur Respir $\mathcal{F}$ 1994;7: 1839-44.

6 Holford N. A size standard for pharmacokinetics. Clin Pharmacokinet 1996;30:329-32.

7 Dahlström K, Larsson P. Lung deposition and systemic availability of budesonide inhaled as nebulised suspension from different nebulisers. Fournal of Aerosol Medicine 1995; 8:79

8 Chua HL, Collis GG, Newbury AM, et al. The influence of age on aerosol deposition in children with cystic fibrosis. Eur Respir f 1994;7:2185-91.

9 Mallol J, Rattray S, Walker G, et al. Aerosol deposition in infants with cystic fibrosis. Pediatr Pulmonol 1996;21:27681 .

10 Everard ML, Hardy JG, Milner AD. Comparison of nebulised aerosol deposition in the lungs of healthy adults following oral and nasal inhalation. Thorax 1993;48:1045- 
11 Wildhaber JH, Devadason SG, Eber E, et al. Aerosol delivery to wheezy infants: a comparison between a nebulizer and two small volume spacers. Pediatr Pulmonol 1997;23: 212-16.

12 Devadason SG, Everard ML, MacErlan C, et al. Lung deposition from the Turbuhaler in children with cystic 97;10:2023-8.

13 Pedersen S, Steffensen G, Ekman I, et al. Pharmacokinetics of budesonide in children with asthma. Eur 7 Clin Pharmacol 1987;31:579-82.

14 Karlsson MO, Bredberg U. Estimation of bioavailability on a single occasion after semisimultaneous drug administration. Pharmacol Res 1989;6:817-21.

15 Bredberg U, Karlsson MO, Borgström L. A comparison between the semisimultaneous and the stable isotope techniques for bioavailability estimation of terbutaline in humans. Clin Pharmacol Ther 1992;52:239-48.

16 Lödrup Carlsen KC, Nikander K, Carlsen KH. How much nebulised budesonide reaches infants and toddlers? Arch Dis Child 1992;67:1077-9.
17 Bisgaard H. Aerosol treatment of young children. Eur Respir Rev 1994;4:15-20.

8 Bisgaard H, Pedersen S, Nikander K. Use of budesonide Turbuhaler in young children suspected of asthma. Eur Respir F 1994;7:740-2.

9 Agertoft L, Pedersen S. Influence of spacer device on drug delivery to young children with asthma. Arch Dis Child 1994;71:217-20.

20 Smaldone GC. Deposition patterns of nebulised drugs: is the pattern important? Fournal of Aerosol Medicine 1994; 7(suppl 1):S25-32.

21 Smaldone GC, Fuhrer J, Steigbigel RT, et al. Factors determining pulmonary deposition of aerosolized pentamidine in patients with human immunodeficiency virus infection. Am Rev Respir Dis 1991;143:727-37.

22 Everard ML, Clark AR, Milner AD. Drug delivery from holding chambers with attached facemask. Arch Dis Child 1992;67:580-5.

23 Agertoft L, Pedersen S. Importance of the inhalation device on the effect of budesonide. Arch Dis Child 1993;69:130-3. 